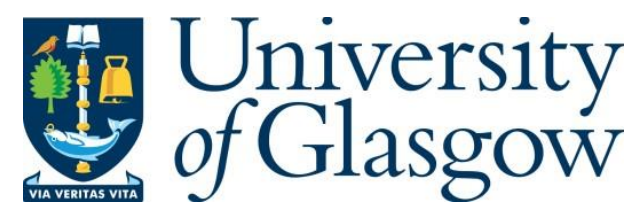

Alabdulqader, E., Lazem, S., Khamis, M. and Dray, S. M. (2018) Exploring

Participatory Design Methods to Engage with Arab Communities. In: CHI EA '18:

36th Annual ACM Conference Extended Abstracts on Human Factors in

Computing Systems, Montréal, QC, Canada, 21-26 Apr 2018, W27. ISBN

9781450356213.

There may be differences between this version and the published version. You are advised to consult the publisher's version if you wish to cite from it.

(C) The Authors 2018. This is the author's version of the work. It is posted here for your personal use. Not for redistribution. The definitive Version of Record was published in the 36th Annual ACM Conference Extended Abstracts on Human Factors in Computing Systems, Montréal, QC, Canada, 21-26 Apr 2018, W27. ISBN 9781450356213 https://doi.org/10.1145/3170427.3170623.

\title{
http://eprints.gla.ac.uk/170207/
}

Deposited on: 5 October 2018

Enlighten - Research publications by members of the University of Glasgow http://eprints.gla.ac.uk 


\section{Exploring Participatory Design Methods to Engage with Arab Communities}

\author{
Ebtisam Alabdulqader \\ Open Lab, Newcastle University \\ Newcastle upon Tyne, UK \\ e.abdulqader1@newcastle.ac.uk \\ Shaimaa Lazem \\ City for Scientific Research and \\ Technology Applications (SRTA-city) \\ Alexandria, Egypt \\ slazem@srtacity.sci.eg \\ Mohamed Khamis \\ Group \\ LMU Munich \\ Mohamed.Khamis@ifi.Imu.de \\ Susan Dray \\ Dray \& Associates \\ USA \\ susan.dray@dray.com
}

Ubiquitous Interactive Systems

Permission to make digital or hard copies of part or all of this work for personal or classroom use is granted without fee provided that copies are not made or distributed for profit or commercial advantage and that copies bear this notice and the full citation on the first page. Copyrights for third-party components of this work must be honored. For all other uses, contact the Owner/Author.

CHI'18 Extended Abstracts, April 21-26, 2018, Montreal, QC, Canada

(c) 2018 Copyright is held by the owner/author(s).
ACM ISBN $978-1-4503-5621-3 / 18 / 04$.

https://doi.org/10.1145/3170427.3170623

\begin{abstract}
ArabHCI is an initiative inaugurated in $\mathrm{CHI} 17$ SIG

Meeting that brought together 45+ HCI Arab and nonArab researchers/practitioners who are

conducting/interested in $\mathrm{HCI}$ within Arab communities. The goal of this workshop is to start dialogs that leverage our "insider" understanding of HCI research in the Arab context and assert our culture identity in design in order to explore challenges and opportunities for future research. In this workshop, we focus on one of the themes that derived our community discussions in most of the held events. We explore the extent to which participatory approaches in the Arab context are culturally and methodologically challenged. Our goal is to bring researchers/practitioners with success and failure stories while designing with Arab communities to discuss methods, share experiences and learned lessons. We plan to share the results of our discussions and research agenda with the wider $\mathrm{CHI}$ community through different social and scholarly channels.
\end{abstract}

\section{Author Keywords}

Arab; Community; Design; Methods; HCI;

\section{ACM Classification Keywords}

H.5.m. Information interfaces and presentation (e.g., $\mathrm{HCI}$ ): Miscellaneous; 


\section{Introduction}

The Arab World, consisting of 22 countries, is rich in its diversity with various religious, ethnic, and linguistic groups sharing the same region. Besides sharing the same language, Arab countries share a strong history dominated by the early colonialism era. Another dominant feature of this region is symbolised in adherence to cultural values that vary from conservative (e.g. Saudi Arabia) to modern and more secular (e.g. Lebanon). Lastly, economic activities and growth influenced the rates and ratio of technology adoption in the region. Since the Arab Spring, there has been a growing interest in the use of technology in the Arab world demonstrated by the role of social media in the Arab uprising. The region came to the forefront once more with the refugee crisis. Many of these studies address Arabs as users of technologies designed in Western countries.

In September 2016, we as a group of Arab HCI researchers started the ArabHCI initiative [4] to express ongoing concern that the majority of research addressing Arab users is conducted from outside the region (i.e., non-Arab countries) and/or is conducted by researchers who are not familiar with its special context. The initiative asserted the need to address the cultural gap between technology designers and users by the locally growing $\mathrm{HCI}$ research and practice communities in the Arab countries. We were clear about adopting an inclusive vision that sees the integration of international (non-Arab) and local expertise significant to advancing $\mathrm{HCI}$ in the Arab region. The first formal ArabHCI meeting was held at CHI 2017 SIG Meeting [3] that was attended by 45+ participants from Arab and non-Arab countries. In the same year, ArabHCI organised a DIS workshop $[2,5]$ with 17+ Arab and non-Arab participants. Additionally, the success of our former events, and their potential to re-shape the HCI in the Arab countries were recently recognized by SIGCHI, who is organising a regional meeting for Arab HCI researchers in January 2018 [17].

The previous ArabHCI meetings aimed broadly to establish local HCI research and practice communities by bringing people together to discuss and review the challenges and opportunities in $\mathrm{HCI}$ education, research, and practice in the Arab context. More specifically, the discussions revolved around main themes proposed by meeting organizers and offered for meetings participants to reflect on. The discussions echoed the issues in the themes, and demonstrated that they are common in the region. Additionally, the discussion reflected the Arab researchers' awareness and willingness to work towards advancing and promoting $\mathrm{HCI}$ in their home countries even if they no longer work there. We realised that the issues are rather difficult to address in one meeting also given the diversity of the research projects and perspectives presented by meetings' participants. Therefore, we propose this workshop to explore in detail one of the challenges that was widely recognized by our participants.

In the following sections, we summarise the emerging themes and discussions as well as expanding on the theme we choose as the focus of this proposal. This workshop theme is the cultural and methodological challenges for engaging Arab communities in participatory design practices.

- HCI in the Arab institutions: The rigid boundaries established between the disciplines of 
engineering and science and of humanities in the culture of higher education result in engineering and science students underrating humanities courses [9], making HCI research homeless. More work is needed to establish partnership with local anthropologists and sociologists. The language is an additional barrier when humanities research is conducted in Arabic language and not necessarily archived online. Research agendas are imposed top-down by the institute and not necessarily reflecting the researchers' interests or supporting for broader engagement with users and communities. In general, funds are scarce when it comes to HCI projects.

- Arab researchers and inclusion: $\mathrm{CHI}$ metrics have shown the limited number of Arab participation in the $\mathrm{CHI}$ conferences in the past four years [7]. This is including the percentage of publication from Arab affiliated researchers and the participation of Arab researchers in the program committees and/or as reviewers. In some countries, many universities do not provide fund for conference publications, since journals are the common appreciated venues for publishing even with a rigorous review process like $\mathrm{CHI}^{\prime}$ s. The ACM new hybrid journal conference submissions would hopefully encourage Arab researchers to submit to the venues adopting them. Some even do not reimburse its staff for ACM or SIGCHI memberships. Though this is also the case in UK and many European countries, these low fees are pretty expensive in local currencies for some Arab countries. Graduate students or post-docs are likely to engage with $\mathrm{CHI}$ community when they are abroad. The rise of islamophobia is another concern for Arabs concerned about travelling abroad.

- Arab context uniqueness: There is a growing body of the research that attempts to address the culture peculiarities in Saudi Arabia $[1,11]$, and study the Arabic users' preferences using Hofstede dimensions $[8,10,13]$. The participants in the ArabHCI DIS 2017 workshop raised the issues of addressing user groups culturally marginalised in the Arab region such as users with disabilities or LGBT $[12,15]$.

- More than UX and Usability: HCI in ICT industry has been focused on addressing usability and user experience, and mostly aims for pleasant graphics design at a late stage in the design process. Discussions showed the need for more user involvement in the design process starting from the early design process stages.

\section{Workshop Theme: Design "with" Arab \\ Communities}

There are many institutional, cultural, and methodological challenges for engaging users in design research. For instance, the lack of centralised ethics

committees and the absence of guarantees for securing collected data along with the concerns that gathered data might be interpreted as culturally or politically inappropriate. Institutional challenges require a change from inside. We take that as a part of the initiative role in helping local researchers to bring awareness about $\mathrm{HCI}$ education and research to Arab institutes. On the other hand, the cultural and methodological challenges are common for local and international researchers who have ongoing studies, or plan to work with Arab 
participants and contexts. Several studies based on the Arab context showed the importance of value sensitive design practices and explored some barriers to involve specific groups of Arab participants $[1,6,11,14,16]$. For instance, culture considerations in recruiting female participants and cross-culture communication with a male researcher have been explored in [11]. The effect of Islamic values on Arabs' use of online romantic relationships and match-making technologies have been researched in $[1,6]$. Design methods such as tangible artifacts and digital storytelling explored and reconfigured to work with women migrants in order to better understand their experiences as refugees [16]. Cultural beliefs and hierarchical structures in refugee families have been studied to identify factors that could inform the design of digital health technologies [14]. We believe more studies are needed to address the appropriateness of Western HCI practices in Arab context. Issues such as the power dynamics if the research team includes non-Arab researchers working with vulnerable communities such as refugees are yet to be fully explored. This workshop will focus on the cultural and methodological barriers to engage participants in design activities starting from the recruitment process to usability evaluation.

\section{Workshop Aims and Outcomes}

This one-day workshop is organised to bring $\mathrm{HCI}$ scholars (Arab and non-Arab) to discuss the participatory design practices and challenges in the Arab context. We aim to better articulate the challenges in applying participatory approaches through scrutinizing research design for successful and failure case studies from the region by experienced researchers. Furthermore, we aim to come up with research agenda to explore issues of participation and engagement in the Arab region. The agenda will address characterizing, evaluating, and/or devising $\mathrm{HCI}$ methods appropriate to the contextual challenges.

Central to this agenda is exploring ways by which Arab $\mathrm{HCI}$ researchers in computing science schools could establish partnerships with local anthropologists and sociologists

Lastly, the workshop will serve a bi-directional goal: to strengthen the community and empower the HCI research in the Arab context, as well as increasing the viability of the Arab researchers and facilitate collaborations. We will invite submissions from researchers and practitioners who attempted participatory approaches in the Arab region. We will ensure a diverse set of attendees including the presence of well-established international HCI research labs in academia and industry to exchange expertise with the Arab researchers and at the same time facilitate future collaboration among HCI researchers, practitioners, and postgraduate students interested in HCI research within the Arab region.

\section{Organizers}

Ebtisam Alabdulqader (main contact) is a Saudi PhD candidate based in Open Lab at Newcastle University, a lecturer in the Information Technology Department at King Saud University (KSU), and affiliated researcher with the Software and Knowledge Engineering Research Group (SKERG). Ebtisam's current research focuses on $\mathrm{HCI}$ aspects of social computing, health informatics, accessibility and mHealth. Her current work is investigating strategies to promote the adoption of digital technologies to augment the current healthcare system and introduce new relational healthcare models. She also led STEM initiative to promote computer science careers in Saudi Arabia. Ebtisam has been 
honored with Teaching and Advising Excellence Award (three times) and the Excellence in Research Award. She is the founder and leader of the ArabHCI initiative and the vice chair for the ACM SIGCHI chapter for Riyadh Saudi Arabia.

Shaimaa Lazem is an Egyptian HCI researcher. She earned her PhD from the Department of Computer Science, Virginia Tech, USA in 2012, and was a postdoctoral at Open Lab, Newcastle University, UK. She then returned to Egypt, where she holds an academic position at the City for Scientific Research and Technology Applications, to establish a research program in HCI. She investigates the use of low-cost technologies and collaborative digital games for teaching mathematics in rural Egypt. She is interested in the theoretical and the empirical research about SelfOrganized Learning Environments as an alternative socio-technical system for children who lack access to qualified Computer Science teachers. In 2017, she was awarded a Newton-Mosharafa (Egypt-UK) grant (The Hilali Network) to promote HCI education in Egypt by exploring flexible learning pedagogical approaches for teaching $\mathrm{HCI}$ to engineering students.

Mohamed Khamis is a PhD student at the Ludwig Maximilians University of Munich (LMU Munich), Germany. His research interests lie generally in ubiquitous computing; in addition to eye tracking and gaze-based interaction, he also does side projects in social drones, the use of electric muscle stimulation in $\mathrm{HCI}$, privacy and usable security. Mohamed started establishing a community of Egyptian HCI researchers in 2014 using a Facebook group. He and other group members planned and organized talks and sessions at the German University in Cairo, Egypt. He organized informal annual gatherings for Egyptian $\mathrm{HCI}$ researchers at the $\mathrm{CHI}$ conference. In $\mathrm{CHI} 2016$, he was among the first to join an initiative for creating a community of ArabHCI researchers. He then actively participated in the first Arab SIG meeting at CHI 2017.

Susan Dray is President of Dray \& Associates, Inc., where she provides contextual and ethnographic user research, usability evaluation, and interface design consultation for a wide range of products systems, and applications. She contributed to the founding of ACM SIGCHI, was the 2006 recipient of the SIGCHI Lifetime Service Award, the 2015 recipient of the SIGCHI Lifetime Achievement in Practice Award and the 2016 recipient of the UXPA's Lifetime Achievement Award. She is also a Fulbright Scholar, an ACM Distinguished Engineer, and a Fellow of the Human Factors and Ergonomics Society.

There is also a Reviewing Committee established to review submitted position papers. Members of the committee are experienced HCI researchers, either based in or familiar with the Arab context. The committee and the workshop organizers will be reviewing the submissions and arranging for the workshop day.

\section{Website and Media Strategy}

An ArabHCI website has been established to promote the community and maximize the presence of the relevant events. Similar to previous events, the workshop's content will be documented in the website, which will includes background information, call for participation, accepted position papers, and reporting the workshop outcomes after the workshop. The website will be maintained after the workshop as a 
focal point for the growing community. Additionally, a social media account has been created on Twitter to encourage community discussions between workshop participants. The website along with social networking accounts will help to publicize the workshop.

$\rightarrow$ Twitter: @arabhci

$\rightarrow$ Website: arabhci.org

$\rightarrow$ Workshop Page: https://arabhci.org/2017/10/chi18-workshop

\section{Pre-Workshop Plans}

The organizers will make an effort to reach a broad audience that might be interested in participating in this workshop. They will publicize the workshop and distribute the call for participation via appropriate professional mailing lists, workshop website, relevant social media accounts, and the existing research communities. Participants will be asked to submit position papers fitting with the workshop goals. Accepted position papers will be posted on the workshop website as open access before the workshop. We aim to recruit 15-25 participants. Experienced senior researchers will be identified and personally invited to join and reflect on the workshop discussions. We aim to invite experienced researchers from both academic and industry backgrounds, who have either conducted or interested in research in Arab context.

\section{Workshop Structure}

The workshop will be conducted over a single day. In the introduction, participants will have a chance to share their personal profiles and expertise. Case studies will be chosen by the workshop organizers based on the submitted contributions for potential attendees. The case studies will be discussed in detail in two panels respectively discussing successful and failed case studies. Each panel consists of the case study researcher(s), who will reflect on their research design and invited senior researchers who will comment and provide feedback. Other participants will contribute to the panel discussions. Workshop participants will be then divided into smaller groups to share their thoughts and come up with a research agenda that focus on further exploring one or more participatory methods for data gathering, community engagement, co-designing, prototyping, etc. Each group will share their agenda and receive peer and expert feedback in the last session. Research agendas and group action plan will be shared on the workshop website.

\section{Workshop Schedule}

09:00 - 10:00 Welcome and Participants Introduction 10:00 - 11:30 Panel: Success stories 11:30 - 12:00 Coffee Break

12:00 - 13:30 Panel: Failure stories

13:30 - 15:00 Lunch Break

15:00 - 16:30 Participatory Challenges (in groups)

16:30 - 17:00 Coffee Break

17:00 - 17:30 Research Agenda Discussion

\section{Post-Workshop Plans}

The workshop discussions will result in the development of a report reflecting the action plan to face the participation challenges and methodological lesson learned while working with Arab context. This also will include developing a research agenda highlighting the research opportunities for testing and developing participatory approaches. In addition to publishing 
these outcomes and the conducted activities in the workshop website, we aim to publish the workshop outcomes and research agenda in the ACM Interactions magazine as well as a special issue of a leading $\mathrm{HCI}$ journal to share workshop participants' research. HCI Arab researchers will be encouraged to discuss the workshop findings, and share it in their local institutions and with their students and relevant research labs. The website and social media account will be maintained as a central channel for communication to spread our outcomes for the larger $\mathrm{HCI}$ community there is an opportunity to learn about the Arab communities. Our aim extends well beyond the workshop setting to include the long-term goal of establishing and empowering a community of HCI researchers conducting research in this context. Furthermore, to promote on-going dialogues between $\mathrm{HCI}$ researchers in Arab countries will be supported by series of events and workshops announced through the website.

\section{Call for Participation (CfP): CHI 2018} Workshop on Exploring Participatory Design Methods to Engage with Arab Communities

This one-day workshop will be organized as part of the CHI 2018, held in Montréal, Canada between 21 and 26 April 2018.

\section{Important Dates}

- Early submission deadline: 5 January 2018

- Early Notification: 20 January 2018

- Final submission deadline: 2 February 2018

- Final Notification: 22 February 2018

ArabHCI is an initiative inaugurated in $\mathrm{CHI} 17$ SIG Meeting that brought together $\mathrm{HCI}$

researchers/practitioners from the Arab World with those who are conducting/interested in research in this context. The goal was to start a community that explores challenges and unique opportunities for future research in Arab countries and with Arab participants. This workshop focuses on one of the themes that derived our community discussions, the extent to which participatory approaches in the Arab context are culturally and methodologically challenged. We invite researchers/practitioners who had success or failure stories designing "with" Arab communities to share their experiences and learned lessons around applying participatory approaches. The workshop will result in developing a research agenda specific to $\mathrm{HCI}$ participatory methods in the Arab world.

Submitted position papers must not exceed four pages in the CHI Extended Abstract Format. The submissions should be sent in PDF format to (arabhci@gmail.com). Position papers will be selected based on the contribution to the workshop theme, quality of presentation, and potential to stimulate discussions. Upon acceptance, at least one of the authors must register for both the workshop and for at least one day of the main conference. For details, please visit: ArabHCI.org

\section{References}

1. Adel Al-dawood, Norah Abokhodair, Houda El Mimouni, and Svetlana Yarosh. 2017. Against Marrying a Stranger: Marital Matchmaking Technologies in Saudi Arabia. In Proceedings of the Conference on Designing Interactive Systems. http://doi.org/10.1145/3064663.3064683

2. Ebtisam Alabdulqader, Norah Abokhodair, and Shaimaa Lazem. 2017. Designing for the arab world. In DIS 2017 Proceedings of the ACM Conference on Designing Interactive Systems, 348-351.

http://doi.org/10.1145/3064857.3064860 
3. Ebtisam Alabdulqader, Norah Abokhodair, and Shaimaa Lazem. 2017. Human-Computer Interaction Across the Arab World. In Proceedings of the $2017 \mathrm{CHI}$ Conference Extended Abstracts on Human Factors in Computing Systems - CHI EA '17, 1356-1359.

http://doi.org/10.1145/3027063.3049280

4. Ebtisam AlAbdulqader and Shaimaa Lazem. 2016. ArabHCI Initiative - Community Website. Retrieved from https://arabhci.org

5. Ebtisam AlAbdulqader, Shaimaa Lazem, and Zohal Azimi. 2017. Design4Arabs Workshop @DIS 2017. ACM Interactions magazine. Retrieved from http://interactions.acm.org/blog/view/design4arab s-workshop-dis-2017

6. Tamara Alsheikh, Jennifer Rode, and Siân Lindley. 2011. (Whose) value-sensitive design: a study of long-distance relationships in an Arabic cultural context. In Proceedings of the ACM 2011 conference on Computer supported cooperative work.

7. KashyapTodi. CHI 2014-2017 Data and Statistics. Retrieved from http://kashyaptodi.com/chi2017

8. Nouf Khashman and Elaine Ménard. 2014. A study of cultural reflection in Egyptian government websites. In Proceedings of the Third International Conference on Design, User Experience, and Usability, 139-147. http://doi.org/10.1007/978-3319-07626-3 13

9. Shaimaa Lazem. 2016. A Case Study for Sensitising Egyptian Engineering Students to UserExperience in Technology Design. In The 7th ACM Symposium on Computing and Development conference (ACM DEV 2016).

10. Aaron Marcus and Sundus Hamoodi. 1986. The Impact of Culture on the Design of Arabic Websites. Proceedings of the 3rd International Conference on Internationalization, Design and Global Development, 10, 8: 386-394.
11. Soud Nassir and Tuck Wah Leong. 2017. Traversing Boundaries: Understanding the Experiences of Ageing Saudis. In Proceedings of the 2017 CHI Conference on Human Factors in Computing Systems, 6386-6397. http://doi.org/10.1145/3025453.3025618

12. Mohammad Othman. Accessibility in the Arab World for People with Disability: Issues and Insights.

13. Ladislao Salmerón, Reem Abu Mallouh, and Yvonne Kammerer. 2017. Location of navigation menus in websites: an experimental study with Arabic users. Universal Access in the Information Society 16, 1: 191-196.

14. Reem Talhouk, Sandra Mesmar, Anja Thieme, et al. 2016. Syrian Refugees and Digital Health in Lebanon: Opportunities for Improving Antenatal Health. Proceedings of the 2016 CHI Conference on Human Factors in Computing Systems: $331-$ 342. http://doi.org/10.1145/2858036.2858331

15. Rina R. Wehbe. Social Media for Change in the Arab World. Retrieved from https://arabhci.org/wpcontent/uploads/2017/05/p10-Rina.pdf

16. Anne Weibert, Konstantin Aal, Nora Oertel Ribeiro, and Volker Wulf. 2017. "This is My Story...": Storytelling with Tangible Artifacts among Migrant Women in Germany. In Companion Publication of the 2017 ACM Conference Companion Publication on Designing Interactive Systems, DIS'17, Edinburgh, United Kingdom, June 10-14, 2017, 144-149. http://doi.org/10.1145/3064857.3079135

17. LAASSLA SIGCHI Meetings - Sponsored by ACM SIGCHI. Retrieved from https://arabhci.org/2017/10/laassla-sigchimeetings/ 